\title{
An adaptive view of non-coding DNA
}

Increasing evidence indicates that although most DNA in eukaryotic genomes is non-coding it is far from being non-functional. An investigation of evolutionary patterns in the Drosophila genome now reinforces this theory by showing that noncoding DNA has been shaped by adaptive evolution - a finding that throws into further doubt the neutral theory of evolution.

By assessing sequence divergence between Drosophila melanogaster and its close relative Drosophila simulans, Peter Andolfatto examined the evolutionary rate of different types of genomic element. This indicated that non-coding regions have evolved more slowly than synonymous sites - those that lead to amino-acid changes in proteins when they are mutated. Polymorphism levels in D. melanogaster are also lower for non-coding sites than for synonymous sites, and together these results provide evidence for selective constraints against mutations in non-coding regions - a sign of functional importance.

Is selective constraint the only force at work in the evolution of non-coding DNA, or might adaptive evolution through positive selection also have a role? Andolfatto tested this on the basis that positive selection leads to increased levels of interspecies sequence divergence relative to levels of polymorphism within species, whereas selective constraint has the opposite effect. In a variation on standard methods, rare polymorphisms were excluded from the analysis, as such variants that have been subject to negative selection can decrease the ability to detect adaptive evolution. Andolfatto found that non-coding sequences showed a higher level of divergence than of polymorphism, with the difference being greater than that seen for synonymous sites - which is evidence that non-coding DNA is subject to adaptive evolution.

The fact that non-coding DNA shows low levels of variation within populations, but has been under positive selection, provides strong support for the theory that such regions have important functional roles. It also suggests that comparative-genomics approaches that are used to identify functional non-coding regions on the basis of sequence conservation are likely to miss many such regions, as they fail to take into account adaptive changes.

Crucially, this study has wider implications for understanding the significance of positive selection, as Andolfatto estimates that many non-coding sites are subject to this evolutionary force. Given the large proportion of eukaryotic DNA that consists of such sites, this presents an important challenge to the widely held theory that it is mainly neutral evolution that shapes eukaryotic genomes.

Louisa Flintoft

(2) References and links ORIGINAL RESEARCH PAPER Andolfatto, P.

Adaptive evolution of non-coding DNA in

Drosophila. Nature 437, 1149-1152 (2005)
FURTHER READING Kondrashov, A. S. Evolutionary biology: Fruitfly genome is not junk. Nature 437, 1106 (2005) WEB SITE

Peter Andolfatto's home page: http://www. biology.ucsd.edu/faculty/andolfatto.html

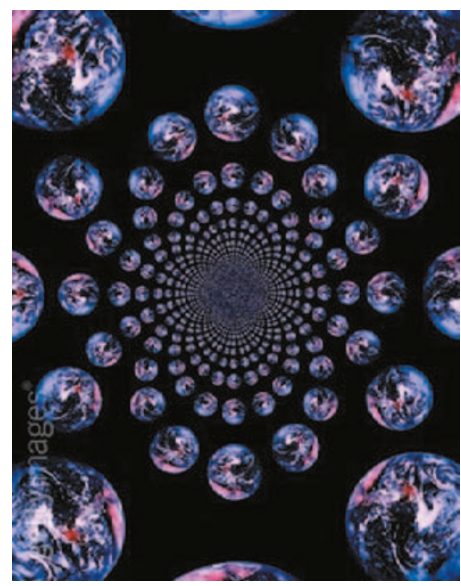

